\title{
The role of LPLP in a changing landscape
}

\author{
François Grin
}

Editor-in-Chief

\section{Linguistic diversity in a new world order}

There seems to be a broad consensus nowadays among scholars, politicians, journalists and citizens at large that in the course of the last few decades, and particularly since the fall of the Berlin wall in 1989, linguistic and cultural diversity has moved to the forefront of public concerns. The prominence of linguistic and cultural diversity as a major social issue of our times is an interesting fact in its own right. Some fifty years ago, technical progress and economic growth were often assumed, particularly among cosmopolitan elites, to usher in a new era in human history, in which matters of language and culture would become little more than peripheral embellishments, or impediments, in the life and progress of modern societies. Despite social change (epitomized, in Europe, by the May 1968 movement) and emerging geopolitical shifts (illustrated by the evolution of the Vietnam war), belief in the unidirectionality of progress remained dominant. In many quarters, languages were essentially seen as problems, and those problems were meant to be solved - for example in the context of decolonization.

However, things have turned out quite differently. Several momentous changes in recent history can help explain why questions of language and culture have regained such visibility. Without attempting an in-depth analysis of these changes and their respective impacts, we can identify three of them.

The first is the geopolitical shift marked by the demise of the erstwhile Soviet Union. A rivalry between major powers that had, for most of the 2oth century, operated as a structuring feature of the international geopolitical order has, though not exactly vanished, fundamentally changed. The dissolution of the Soviet Union at the turn of the 199os has allowed the re-emergence and re-assertion of components of ethnic, linguistic and cultural diversity that had often been discounted as passé or irrelevant. This re-emergence bears witness to the remarkable resilience of the linguistic and cultural facets of human societies.

The second of these changes is embodied in accelerated technical progress, particularly in ICT, including automatic translation, together with the possible practical uses of these technical advances. The effects on diversity of technical 
development are ambiguous. On the one hand, technical progress expands the possibilities of worldwide uniformisation, whether in terms of symbolic references, modes of material consumption or, of course, linguistic and cultural practices. On the other hand, it also multiplies the ways in which such practices can manifest themselves. Technology facilitates the use of a wider range of languages (including languages with a very small number of speakers, as well as languages that are in a minority situation in context $X$ despite being a majority language in some other context $Y$ ). They can be used in private, public or statal contexts more cheaply and conveniently; the palette of languages that we are liable to meet in our daily lives is tendentially larger, even if, at the same time, numerous languages are falling into disuse, mainly because of failing intergenerational transmission.

The third of these changes is commonly referred to as "globalisation". Although a fully satisfactory definition of globalisation probably still needs to be proposed (a challenge in itself, given that globalisation is currently unfolding before our eyes), many of its key features can be relatively easily pinpointed: the spread (notwithstanding setbacks in various countries) of certain social, political and economic modes of societal organisation and the associated institutions such as market capitalism and representative democracy; lower barriers to trade and a concomitant increase of the share of traded goods and services in the world economy; a significantly heightened mobility of people, giving rise to more varied and ubiquitous forms of permanent or temporary migration.

Although these three trends (geopolitical upheavals, technological progress, and globalisation) are complex and their interplay only partly understood, it is plausible to view them as hallmarks of substantial changes in the world order, with implications that are still difficult to predict. One of these implications, however, is that the diversity of languages and cultures - issues that had largely been eclipsed since the end of the Second World War in scientific, political and media discourse - have been brought back to centre stage. This reassertion is reflected in a wide panoply of phenomena which can be approached under a variety of analytical angles. The ways in which languages mesh with the construction of individual identity, as well as their role in creating a sense of belonging to a community, are shifting. These changes are not always tension-free, and an increasingly banal interaction with linguistic otherness, particularly in the urban centres of prosperous countries, does not exempt modern societies from bouts of nativism - possibly in reaction to the former phenomenon.

These changes also carry consequences for scientific research in the social sciences and humanities. And this, in turn, raises the question of the role of a journal such as Language Problems and Language Planning (LPLP). 


\section{Complexity and interdisciplinarity}

First published under its current name in $1977, L P L P$ is the heir of the erstwhile La monda lingvo-problemo, established in 1969. Rather than a refoundation, the change of name reflected continuity in the journal's topic and concerns. This makes it, by a long shot, the oldest of the scientific journals in language policy. Historically, it was the first to address language, multilingualism and linguistic diversity not just as a set of phenomena to be described and accounted for, but as a feature of human societies that the latter may legitimately seek to influence through deliberate action - that is to say, through public policies. This focus, in many ways, was a prescient one: the advent of the three major trends just sketched out has resulted in more complex linguistic environments; the understanding of the processes that generate them, as well as of the conduits through which human societies can try and steer their linguistic environments in certain directions deemed desirable, have also gained in strategic importance. The "linguistic human rights" approach, which dovetails with a public policy perspective in seeking to establish greater fairness between individuals and groups defined in terms of their linguistic attributes, is one illustration of the heightened importance - and convergence - of these concerns.

"Complexity" is not merely a generic catchphrase: the term harks back to an approach that pays attention to features such as non-linearity, power laws, spontaneous order, etc., calling for a careful systemic approach to the processes in question. Awareness of complexity does not necessarily require engaging in the formal modelling of such features, but it certainly means devoting sustained attention to the interconnection between the different facets of a process and to the interplay between micro-, meso- and macro-level developments. Such attention quite naturally builds on normal scientific concerns, such as distinguishing between the unique or, on the contrary, generalizable character of a phenomenon. Awareness of complexity is fully in line with an epistemological tradition concerned with consistency between theoretical analysis and empirical observation. In this first sense, attention to the complexity of language-related processes (in policy intervention as well as in the broader social, political, historical, cultural, and economic context that surrounds and precedes it) must be a priority of the work that LPLP will seek to publish and support.

Complexity also carries another implication with major importance for this journal, namely, the need to recognise how crucial it is to avoid silo thinking, and how indispensable it is to cooperate across disciplines. Language problems and the language policies designed to address them are not just linguistic, let alone sociolinguistic questions. They are questions involving the learning, knowledge and use of languages across domains and facets of human experience, as well as the 
associated representations carried through various genres of discourse. The language disciplines, of course, are centrally placed to offer concepts and tools in order to study these questions, but the latter also have social, political, economic, geographical, legal, educational, and psychological dimensions. The implication is straightforward: the study of language problems and language planning must be interdisciplinary, and interdisciplinarity will remain a compass for this journal.

We wish $L P L P$ to reinforce its position as a scientific forum on language problems and language planning for scholars from across the entire spectrum of the social sciences and humanities; the red thread running through the contributions by the scientific community whose work is published in LPLP ought to be not a similarity in training or disciplinary background - let alone affiliation to a particular chapel with its liturgy and dogma - but a shared concern, an interest, a fascination, or perhaps even a passion, for language and languages, as well as their role in modern societies and institutions. This common ground transcends the disciplinary perspectives that may be brought to bear on language issues, and one of the principles inspiring the editorship of LPLP is that a deliberate effort to combine the conceptual or methodological inputs of one's discipline with some of the inputs proposed by other disciplines is among the most exciting and stimulating adventures that research has to offer.

\section{About "problems", "planning", and research epistemology}

As noted above, LPLP has been around for several decades, and perhaps its (unchanged) name requires a few words of commentary in a contemporary (and changed) context.

The reference to "language problems" must not be misunderstood or construed into something that it does not mean. The term "problem" does not carry any negative assumption. Quite simply, "language-in-society" (to use a hyphenated expression attributed to Joshua Fishman), whether in terms of learning, use, dissemination, transmission, etc., confronts actors with various difficulties and challenges. It is often linked up with the production, reproduction and allocation of material and symbolic resources, involving the (usually unequal) distribution of economic, social, political and cultural influence. Even more simply, language-insociety raises questions which actors have to answer, individually or collectively. This is what we mean by "language problems" - nothing more, also bearing in mind that the very identification of what is identified as a "problem" requiring treatment through policy and planning is, in itself, a process that deserves critical examination. In this sense, all scientific inquiry worth its salt, in the social sciences, is inherently critical. 
The term "language planning" in the journal's title bears witness to its history, and in particular to some of the issues at the forefront of research concerns in the 1970s, including decolonisation. At that time, even if political and constitutional change had largely been accomplished in the erstwhile colonial empires, much remained to be done in order for decolonisation to materialise, not only in formal state structures, but in other aspects of life as well. Language pervaded them all, raising the question, among others, of the choice of language (or languages) to be used in government, public administration, education systems and in the media. In this context, some of the questions that arose could involve direct intervention on the corpus of certain languages, an endeavour traditionally described by the phrase language planning. Alternatively, instead of implying a reference to matters of corpus, "planning" has been used to highlight the need for a "plan" when implementing language policies. It goes without saying that the use of the word "planning" in the journal's title should not be interpreted as a restriction to this set of issues. Should a change of name be considered (but we do not see it as necessary), we might replace the word "planning" by the word "policy", itself understood in a broad sense and extending to the study of governance and governmentality. We could also say that the presence of the word "planning" in the journal's name is a historically explainable stand-in for "policy", and that our focus on policy allows for a natural connection with the politics in which policies unfold.

Beyond an array of terms (planning, policy, politics, governance, governmentality), what matters is the thematic focus on deliberate action at the societal level - including the institutions emerging from a society. This action results from the confrontation of goals and constraints on available resources, but it goes without saying that the identification and the setting of some policy goals, as opposed to alternative goals, as well as the mobilisation and use of some resources in order to achieve these goals, reflects a certain state of affairs. A key feature of the latter is the distribution of material and symbolic resources among social actors, in a certain context at a certain time. This is why LPLP intends to emphasise the relevance not just of the effectiveness and cost-effectiveness of language policies, but also of their fairness. How we respond to language problems through policy has implications for the more or less fair distribution as well as for the more or less efficient allocation of these resources. By shedding light on these processes, research in language issues contributes not only to scientific knowledge, but also to democratic debate. It serves to make the democratic debate a better-informed one, and this applies to policies derived from political programmes initiated at central governmental level, as well as to policies stemming from grassroots initiatives, and then turned into formal public policies after having been endorsed by local or national authorities and integrated into a legal-institutional framework. Typically, all policies involve top-down and bottom-up features, and the study of 
language policies also serves to identify expressions of both, along with the interplay between them. In sum, the perspective just described reflects the conviction that language problems, as well as the responses to those problems through policies, necessarily involve allocative and distributive dimensions - and hence issues of power. The identification of both types of phenomenon is a relevant and necessary endeavour.

The identification of such phenomena in a particular case, however, requires more than the claim that they exist. Ascribing a particular interpretation to a set of observations can only be convincing if its plausibility is established through various means. In other words, falsifiability remains a crucial quality criterion, to which this journal will remain alert. Of course, very valuable heuristic insights can be obtained through interpretive methods rooted in hermeneutics; and falsifiability does not necessarily require an analysis to be buttressed by a formal model or statistical tests. However, the study of language problems and language planning needs to rest on more than the mere assertion of one interpretation. Conceptual precision and empirical rigour are required in order to avoid tendentious overinterpretation, a risk to which scholarly discourse on language policy is not immune.

\section{Language policies and scale}

Our discussion so far has emphasised several editorial priorities : (i) the need to be aware of complexity in language processes; (ii) the relevance of interdisciplinarity, where contributors from various disciplines are bound by a shared passion for the study of language and linguistic diversity; (iii) the attention that needs to be paid to the joint presence of allocative and distributive dimensions (how a language policy process operates, but also what redistribution of material and symbolic resources ensue from it); (iv) the importance of classic scientific standards of analytical rigour.

These four priorities all refer to ways of doing research and to the spirit in which language problems and language planning may be approached. One separate point, however, needs to be made regarding topics that are of particular interest to this journal. It has to do with scale. LPLP has a solid tradition of interest in relatively macro-level questions - what informal everyday speech calls "largescale" issues, even if, in the arithmetic of cartography, they are actually described as small-scale. LPLP is particularly interested in the rigorous investigation of "big" language problems, including those that arise at the level of the world as a whole: for example, what elements of explanation can we propose to better understand patterns of language spread and language decline? How, and under what condi- 
tions, can these patterns be influenced through policy? What role might lingue franche play in this context? Our interest in the big picture also explains why the theme of interlinguistics will keep enjoying visibility in this journal, which is one of the few to have consistently devoted space to it throughout the years.

In closing, and as I take up my duties as editor-in-chief of $L P L P$, I wish to express thanks, on my own behalf and on behalf of the journal, to all those who have served it so well for many years. This includes the former editorial team, as well as colleagues who are now leaving the Board and others who are staying on. My gratitude extends to those who are joining it now. Readers will find the list of Board and editorial team members on the first page of the first 2019 issue (Vol. 43, No. 1), and will notice its reinforced interdisciplinary orientation, with colleagues from various specializations in linguistics, but also from political science, sociology, the education sciences, economics and law. I am immensely fortunate in being able to count on the support of these eminent colleagues and friends, and will do my utmost to prove worthy of their trust in my new editorial duties.

François Grin Geneva, 4 December 2018 